Proceeding Series of the Brazilian Society of Computational and Applied Mathematics

\title{
Comparative Study of four Generalized Predictive Controllers for Reference Tracking and Disturbance Attenuation
}

\author{
Rejane de Barros Araújo ${ }^{1}$ \\ Daniel Cavalcanti Jeronymo ${ }^{2}$ \\ Antonio Augusto Rodrigues Coelho ${ }^{3}$ \\ Federal University of Santa Catarina \\ Department of Automation and Systems 88040-900 \\ Florianopolis, SC, Brazil
}

\begin{abstract}
The purpose of this paper is first to review the standard Generalized Predictive Controller (GPC) design, second, to establish a comparative study between the GPC with a prefilter $T_{f}\left(q^{-1}\right)$, the GPC with a parameter of the future reference trajectory and the GPC that includes a positional model but with a integral polynomial weighing factor for the error. Simulation results are shown and discussed.
\end{abstract}

Keywords. predictive control, dynamic stability, control system design, target tracking.

\section{Introduction}

The Generalized Predictive Controller (GPC) has been successfully implemented in several applications and is still under investigation [1-4]. Over the last decades different design formalisms have been developed with the aim to guarantee closed-loop dynamic aspects like performance, stability, robustness and input constraints.

The purpose of this paper is to review the standard GPC design of [1] and to establish a comparative study between T-GPC, GPC with a filter $T_{f}\left(q^{-1}\right) \neq 1$ [2], FR-GPC (Filter Reference GPC), GPC with a parameter of the future reference trajectory [5] and FP-GPC (Filter Positional GPC), GPC that uses a positional model but with integral polynomial weighing factor for reference and output signals [6].

The idea is to investigate how the GPC filtered affect the performance of the closed-loop system in terms of reference tracking, disturbance attenuation, stability and robustness. Additionally, to show the capacity of the FP-GPC in dealing with setpoint tracking and disturbance rejection with very satisfactory performance between GPC controllers (standard GPC, T-GPC and FR-GPC). Numerical essay demonstrates the effectiveness of the GPC control algorithms and performance indicators are shown.

\footnotetext{
${ }^{1}$ rejane.barros@posgrad.ufsc.br

2d.cavalcanti@posgrad.ufsc.br

antonio.arc@ufsc.br
} 
This paper is organized as follows. Sections 2, 3, 4 and 5 present briefly each control design for the GPC, T-GPC, FR-GPC and FP-GPC, respectively. Section 6 describes a comparative study between the controllers, examining aspects such as transfer function, sensitivity function and control law. Section 7 shows a numerical simulation. Finally, conclusions are given in Section 8.

\section{Standard GPC Design}

The standard GPC design proposed in [1] can be derived by the following discrete transfer function on the CARIMA model (Controlled Auto-Regressive Integrated Moving Average):

$$
A\left(q^{-1}\right) y(t)=q^{-d} B\left(q^{-1}\right) u(t-1)+T_{f}\left(q^{-1}\right) \frac{e(t)}{\Delta},
$$

where $y(t)$ is the process output, $u(t)$ is the control signal, $e(t)$ is the zero mean white noise, $d$ is the dead-time, $T_{f}\left(q^{-1}\right)=1$ and $\Delta=\left(1-q^{-1}\right)$. The roots of the polynomials $A\left(q^{-1}\right)$ and $B\left(q^{-1}\right)$ are the open-loop poles and zeros, respectively.

The standard GPC control law is obtained by minimizing the cost function given by

$$
J=\sum_{j=1}^{N_{y}} \delta[\hat{y}(t+j / t)-w(t+j)]^{2}+\lambda \sum_{j=1}^{N_{u}}[\Delta u(t+j-1)]^{2},
$$

where $w(t)$ is the setpoint, $\delta$ and $\lambda$ are error and control weighting, respectively, $N_{y}$ is the output prediction horizon and $N_{u}$ is the control horizon. From minimization of the cost function (2), the control law of the unconstrained GPC is described by

$$
\Delta u(t)=K_{G P C}(w-f)=\sum_{j=1}^{N_{y}} k_{j}[w(t+j)-f(t+j)],
$$

where $K_{G P C}$ is the first row of the matrix $\left(G^{T} G+\lambda I\right)^{-1} G^{T}, f$ is the free response, $w$ is the reference. Using the GPC design, defined in (3), then the RST canonical form of the controller is written as

$$
R\left(q^{-1}\right) \Delta u(t)=T\left(q^{-1}\right) w(t)-S\left(q^{-1}\right) y(t) .
$$

The equation (4) represents the polynomial control structure of two degree of freedom, where $R\left(q^{-1}\right), S\left(q^{-1}\right)$ and $T\left(q^{-1}\right)$ are polynomials and obtained from (3). The RST canonical form have filters in the reference represented by $T\left(q^{-1}\right)$, in the output represented by $S\left(q^{-1}\right)$ and in the error signal represented by $1 / \Delta R\left(q^{-1}\right)$ [7]. The block diagram of the RST structure from the GPC design is shown in Figure (1).

\section{T-GPC Design}

Now, consider the equation (1) where $T_{f}\left(q^{-1}\right)$ is a polynomial which implements a prefilter as follows $\left(1-\beta q^{-1}\right)$. The T-GPC control algorithm is obtained by minimizing 


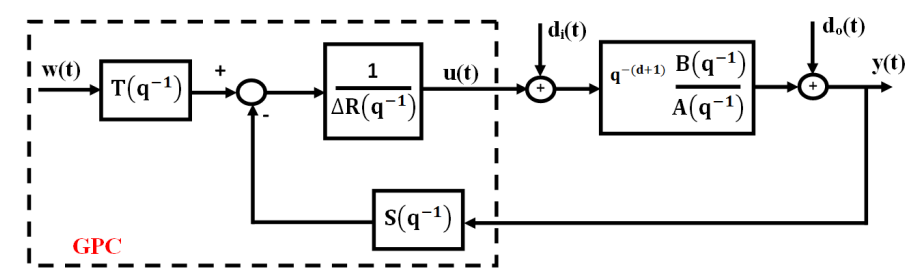

Figure 1: Polynomial RST control structure for GPC design.

the cost function of equation (2). The control law is equivalent from the standard GPC design (exhibits differences in the free response and GPC gain $\left(K_{G P C}\right)$ ) and is calculated as follows:

$$
\Delta u(t)=\tilde{K}_{G P C}(w-\tilde{f})=\tilde{K}_{G P C}\left(w(t)-\frac{\tilde{F}_{j}\left(q^{-1}\right)}{T_{f}\left(q^{-1)}\right.} y(t)-\frac{\tilde{H}_{j}\left(q^{-1}\right)}{T_{f}\left(q^{-1)}\right.} \Delta u(t-1)\right) .
$$

Diophantine equations, for the T-GPC synthesis, are solved using $T_{f}\left(q^{-1}\right)=\left(1-\beta q^{-1}\right)$, then modifying the design matrices $\widetilde{G}, \widetilde{H}, \widetilde{F}$ and, consequently, the polynomials $R\left(q^{-1}\right)$, $S\left(q^{-1}\right)$ and $T\left(q^{-1}\right)$.

\section{FR-GPC Design}

Assuming equation (1) with $T_{f}\left(q^{-1}\right)=1$, the FR-GPC control law is given by minimizing the cost function of equation (2), where the future reference $w(t+j)$ is calculated by the following equation $[1,5]$ :

$$
w(t)=y(t), \quad w(t+j)=(1-\gamma) r(t)+\gamma w(t+j-1),
$$

where $0 \leq \gamma<1$ and $r(t)$ is the setpoint. To the future term $T\left(q^{-1}\right) w(t+j)$ of the standard GPC law, the future reference is replaced by

$$
w(t+j)=\gamma^{j} y(t)+\left(1-\gamma^{j}\right) r(t) .
$$

Then, the future term can be represented as follows:

$$
T\left(q^{-1}\right) w(t+j)=t_{r} r(t)+t_{y} y(t), \quad t_{r}=\sum_{j=1}^{N_{y}}\left(1-\gamma^{j}\right) k_{j}, \quad t_{y}=\sum_{j=1}^{N_{y}} \gamma^{j} k_{j} .
$$

The FR-GPC control law is written as

$$
\Delta u(t)=K_{G P C}\left(\left(1-\gamma^{j}\right) w-f-\gamma^{j} y\right) .
$$




\section{FP-GPC Design}

Consider the deterministic CAR model (Controlled Auto-Regressive) of the controlled plant characterized by the following positional discrete transfer function:

$$
A\left(q^{-1}\right) y(t)=q^{-d} B\left(q^{-1}\right) u(t-1) .
$$

The GPC control law is obtained by minimizing the cost function of the form

$$
J=\sum_{j=1}^{N_{y}}\left\{\phi_{y}(t+j)-\phi_{w}(t+j)\right\}^{2}+\lambda \sum_{j=1}^{N_{u}} u^{2}(t+j-d-1),
$$

with $\phi_{y}(t+j)$ and $\phi_{w}(t+j)$ auxiliary output and reference variables and are defined as

$$
\phi_{y}(t)=P\left(q^{-1}\right) y(t)=\frac{K_{\alpha} \alpha\left(q^{-1}\right)}{\Delta} y(t), \quad \phi_{w}(t)=P\left(q^{-1}\right) w(t)=\frac{K_{\alpha} \alpha\left(q^{-1}\right)}{\Delta} w(t) .
$$

The term $\phi_{y}(t+j)$ is replaced by the estimated value with the equation (10) multiplied by $P\left(q^{-1}\right)$ and it can be rewritten as

$$
\Delta A\left(q^{-1}\right) \phi_{y}(t+j)=K_{\alpha} \alpha\left(q^{-1}\right) B\left(q^{-1}\right) u(t+j-d-1) .
$$

Then, the minimization of the cost function of the FP-GPC for the unconstrained case, generates the control vector and is calculated by

$$
u(t)=K_{G P C}\left[\Phi_{w}(t)-\Phi_{f}(t)\right],
$$

which is the similar notation of the incremental GPC design proposed by [1] for the incremental fixed structure. It is noteworthy that the FP-GPC design is an alternative synthesis to the standard formalism of the GPC and more details of the FP-GPC design can be found in $[6]$.

\section{Comparative Analysis of GPC Controllers}

This section shows a comparative study between standard GPC [1], T-GPC $[2,3]$, FR-GPC [1,5] and FP-GPC [6] control algorithms, in order to evaluate the feasibility of these controllers regarding the performance and robustness to treat reference tracking, disturbance attenuation and model-plant mismatch.

The block diagram of Figure (1) can be used for analysis of the four controllers on the RST structure with changes on polynomials $R\left(q^{-1}\right), S\left(q^{-1}\right), T\left(q^{-1}\right)$ for each controller. For the case of the T-GPC and FP-GPC there is the inclusion of the $T_{f}\left(q^{-1}\right)$ and $K_{\alpha} \alpha\left(q^{-1}\right)$ filters, respectively. Table (1) shows the design equations of the polynomials $R\left(q^{-1}\right)$, $S\left(q^{-1}\right), T\left(q^{-1}\right)$ for GPC controllers.

From the closed-loop transfer function, sensitivity function and control law, as shown in Table (2), it is possible to make the following observations: 
Table 1: Comparative equations of standard GPC, T-GPC, FR-GPC and FP-GPC.

\begin{tabular}{|c|c|c|c|}
\hline & $R\left(q^{-1}\right)$ & $S\left(q^{-1}\right)$ & $T\left(q^{-1}\right)$ \\
\hline GPC & {$\left[1+q^{-1} \sum_{j=1}^{N_{y}} k_{j} H_{j}\right]$} & $\sum_{j=1}^{N_{y}} k_{j} F_{j}$ & $\sum_{j=1}^{N_{y}} k_{j} q^{j}$ \\
\hline T-GPC & $\frac{T_{f}\left(q^{-1}\right)+q^{-1} \sum_{j=1}^{N_{y}} k_{j} \tilde{H}_{j}\left(q^{-1}\right)}{T_{f}\left(q^{-1}\right)}$ & $\sum_{j=1}^{N_{y}} \frac{k_{j} \widetilde{F}_{j}}{T_{f}\left(q^{-1}\right)}$ & $\sum_{j=1}^{N_{y}} k_{j} q^{j}$ \\
\hline FR-GPC & $1+q^{-1} \sum_{j=1}^{N_{y}} k_{j} H_{j}$ & $\sum_{j=1}^{N_{y}} k_{j}\left(F_{j}-\gamma^{j}\right)$ & $\sum_{j=1}^{N_{y}}\left(1-\gamma^{j}\right) k_{j} q^{j}$ \\
\hline FP-GPC & {$\left[1+q^{-1} \sum_{j=1}^{N_{y}} k_{j} \bar{H}_{j}\right] u(t)$} & $\sum_{j=1}^{N_{y}} k_{j} F_{j} K_{\alpha} \alpha\left(q^{-1}\right)$ & $\sum_{j=1}^{N_{y}} k_{j} q^{j} K_{\alpha} \alpha\left(q^{-1}\right)$ \\
\hline
\end{tabular}

Table 2: Comparative transfer functions of GPC, T-GPC, FR-GPC and FP-GPC.

\begin{tabular}{|c|c|}
\hline - & Transfer Function \\
\hline \multirow{3}{*}{ Standard GPC } & $y(t)=\frac{T\left(q^{-1}\right) B\left(q^{-1}\right)}{A\left(q^{-1}\right) \Delta R\left(q^{-1}\right)+B\left(q^{-1}\right) S\left(q^{-1}\right)} w(t-d)$ \\
\hline & $S=\frac{A\left(q^{-1}\right) \Delta R\left(q^{-1}\right)}{A\left(q^{-1}\right) \Delta R\left(q^{-1}\right)+B\left(q^{-1}\right) S\left(q^{-1}\right)}$ \\
\hline & $u(t)=\frac{1}{\Delta R\left(q^{-1}\right)}\left[T\left(q^{-1}\right) w(t)-S\left(q^{-1}\right) y(t)\right]$ \\
\hline \multirow{3}{*}{ T-GPC } & $y(t)=\frac{T_{f}\left(q^{-1}\right) B\left(q^{-1}\right) T\left(q^{-1}\right)}{\left[A\left(q^{-1}\right) \Delta R\left(q^{-1}\right)+B\left(q^{-1}\right) S\left(q^{-1}\right)\right] T_{f}\left(q^{-1}\right)} w(t-d)$ \\
\hline & $S=\frac{A\left(q^{-1}\right) \Delta \widetilde{R}\left(q^{-1}\right)}{\left[A\left(q^{-1}\right) \Delta R\left(q^{-1}\right)+B\left(q^{-1}\right) S\left(q^{-1}\right)\right] T_{f}\left(q^{-1}\right)}$ \\
\hline & $u(t)=\frac{T_{f}\left(q^{-1}\right)}{\Delta \widetilde{R}\left(q^{-1}\right)}\left[T\left(q^{-1}\right) w(t)-\frac{\widetilde{S}\left(q^{-1}\right)}{T_{f}\left(q^{-1}\right)} y(t)\right]$ \\
\hline \multirow{3}{*}{ FR-GPC } & $y(t)=\frac{B\left(q^{-1}\right) t_{r}\left(q^{-1}\right)}{A\left(q^{-1}\right) \Delta R\left(q^{-1}\right)+B\left(q^{-1}\right)\left(S\left(q^{-1}\right)-t_{y}\left(q^{-1}\right)\right)} w(t-d)$ \\
\hline & $S=\frac{A\left(q^{-1}\right) \Delta R\left(q^{-1}\right)}{A\left(q^{-1}\right) \Delta R\left(q^{-1}\right)+B\left(q^{-1}\right)\left(S\left(q^{-1}\right)-t_{y}\left(q^{-1}\right)\right)}$ \\
\hline & $u(t)=\frac{1}{\Delta R\left(q^{-1}\right)}\left[t_{r}\left(q^{-1}\right) w(t)-\left(S\left(q^{-1}\right)-t_{y}\left(q^{-1}\right)\right) y(t)\right]$ \\
\hline \multirow{3}{*}{ FP-GPC } & $y(t)=\frac{K_{\alpha} \alpha\left(q^{-1}\right) B\left(q^{-1}\right) T\left(q^{-1}\right)}{A\left(q^{-1}\right) \Delta \bar{R}\left(q^{-1}\right)+K_{\alpha} \alpha\left(q^{-1}\right) B\left(q^{-1}\right) S\left(q^{-1}\right)} w(t-d)$ \\
\hline & $S=\frac{A\left(q^{-1}\right) \Delta \bar{R}\left(q^{-1}\right)}{A\left(q^{-1}\right) \Delta \bar{R}\left(q^{-1}\right)+K_{\alpha} \alpha\left(q^{-1}\right) B\left(q^{-1}\right) S\left(q^{-1}\right)}$ \\
\hline & $u(t)=\frac{K_{\alpha} \alpha\left(q^{-1}\right)}{\Delta \bar{R}\left(q^{-1}\right)}\left[T\left(q^{-1}\right) w(t)-S\left(q^{-1}\right)\right]$ \\
\hline
\end{tabular}

T-GPC, FR-GPC and FP-GPC controllers are equivalent, but with different features, according to design filters $T_{f}\left(q^{-1}\right), t_{y}\left(q^{-1}\right), t_{r}\left(q^{-1}\right)$ and $K_{\alpha} \alpha\left(q^{-1}\right)$ for each control loop.

For the T-GPC case, the two degree of freedom structure can be exploited only for disturbance attenuation which depends on $T_{f}\left(q^{-1}\right)$, however the reference tracking is affected by the calibration of the GPC parameters.

In the case of the FR-GPC and FP-GPC, both reference tracking and disturbance rejection can be exploited by the filters $t_{y}\left(q^{-1}\right), t_{r}\left(q^{-1}\right)$ and $K_{\alpha} \alpha\left(q^{-1}\right)$, respectively.

On the other hand, the prefilter $T_{f}\left(q^{-1}\right)$ does not modify the closed-loop poles and control law in the T-GPC, however, $t_{y}\left(q^{-1}\right)$ and $K_{\alpha} \alpha\left(q^{-1}\right)$ changes the robustness, closedloop stability and control law in FR-GPC and FP-GPC controller designs. 


\section{Simulation Results}

This essay is based on a numerical simulation of a multiple poles system described in [5]. Plant and model transfer functions are given by

$$
G_{p}(s)=\frac{1}{(s+1)^{8}}, \quad G_{m}(s)=\frac{0.007}{\left(s^{2}+0.73 s+0.1\right)} .
$$

Figure (2) shows the closed-loop responses for the GPC controllers, using step setpoint changes and a constant disturbance on the plant output (10\% of the setpoint magnitude value at time $t=300 \mathrm{~s}$ ). The controllers have equivalent dynamic behavior, but the FP-GPC has better reference tracking and disturbance attenuation performance than the others controllers with small control variance and low output oscillation. The tuning parameters of the controllers are $N_{y}=4, N_{u}=1, \lambda=0.5$ and sampling period of $1 \mathrm{~s}$.
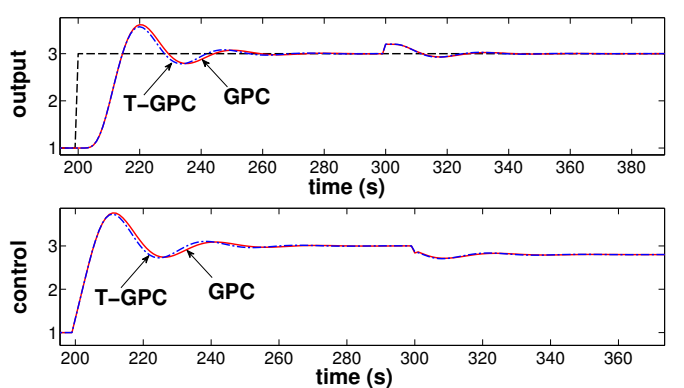

(a) Standard GPC and T-GPC
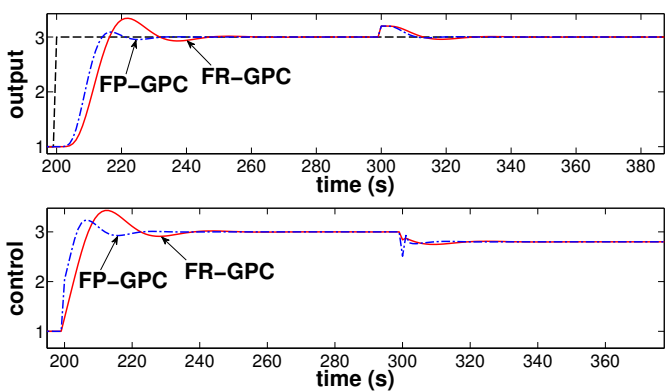

(b) FR-GPC and FP-GPC

Figure 2: System response to the GPC, T-GPC, FR-GPC and FP-GPC.

Filter tuning parameters of each controller are selected from different ways. For the T-GPC, the polynomial $T_{f}\left(q^{-1}\right)$ is tuning using the guidelines suggested in [2], where the time constant of the filter lies in the neighborhood of the dominant poles of $A\left(q^{-1}\right)$. For the FR-GPC, the parameter $\gamma$ is the same used in [5]. For the case of the FP-GPC, the filter parameters $\left(K_{\alpha}\right.$ and $\left.\alpha\left(q^{-1}\right)\right)$ are calibrated through a multi-objective optimization algorithm based on the sensitivity function and Integrated Absolute Error (IAE) criterion.

Table (3) shows the filter parameters for each GPC design and the performance indeces (IAE and TVC - Total Variation of Control) of the four controllers. We can observed that the FP-GPC performance is better than the others controllers (smaller values of IAE and TVC).

\section{Conclusions}

This paper has investigated a comparative study between the standard GPC, T-GPC, FR-GPC and FP-GPC controllers, analyzing how the filter tuning parameters are affecting the performance of the closed-loop system, evaluating reference tracking, disturbance attenuation and stability conditions. 
Table 3: Tuning parameters and IAE, TVC criteria.

\begin{tabular}{|c|c|c|c|}
\hline & Filter Values & IAE & TVC \\
\hline GPC & - & 17.6382 & 9.1361 \\
\hline T-GPC & $T_{f}\left(q^{-1}\right)=\left(1-0.45 q^{-1}\right)$ & 17.2660 & 9.2079 \\
\hline FR-GPC & $\gamma=0.69$ & 15.7137 & 6.5636 \\
\hline FP-GPC & $K_{\alpha} \alpha\left(q^{-1}\right)=2.4375\left(1-0.7294 q^{-1}\right)$ & $\mathbf{1 1 . 1 8 6 3}$ & $\mathbf{6 . 4 1 6 0}$ \\
\hline
\end{tabular}

Initially, the paper described briefly the control design for the GPC, indicating the process model, cost function and control law, achieving the RST canonical form of the controller. Next, it was presented a comparative analyses of the polynomials $R\left(q^{-1}\right)$, $S\left(q^{-1}\right), T\left(q^{-1}\right)$ and the closed-loop transfer function, sensitivity function and control law for each controller, highlighting the influence of the filter design on the closed-loop polynomials and, consequently, on the performance and robustness of the system.

Finally, a numerical simulation was shown to a multiple poles system with model-plant mismatch to assess not only the stability, performance and robustness of GPC controllers for setpoint tracking and disturbance rejection, but also to demonstrate that the FP-GPC has performed best performance than the others GPC controllers.

\section{References}

[1] D. W. Clarke; C. Mohtadi and P. S. Tuffs, Generalized predictive control - Part I: the basic algorithm, Automatica, pp. 137-148, (1987).

[2] J. A. Rossiter, Model-based predictive control: a practical approach, CRC Press LLC, ISBN: 0-8493-1291-4, (2004).

[3] E. F. Camacho and C. Bordons, Model predictive control, Springer-Verlag, London, (2004).

[4] D. Q. Mayne, Model predictive control: recent developments and future promise, Automatica, pp. 2967-2986, (2014).

[5] T. Sato and A. Inoue, Improvement of tracking performance in self-tuning PID controller based on generalized predictive control, International Journal of Innovative Computing, Information and Control, pp. 491-503, (2006).

[6] R. B. Araújo; D. C. Jeronymo and A. A. R. Coelho, Incremental and positional generalized predictive controller for offset free reference tracking, Brazilian Congress of Automatic Control, Brazil, (2014).

[7] I. D. Landau, The RST digital controller design and applications, Control Engineering Practice, pp. 155-165, (1998). 